Revista Iberoamericana, Vol. LXXIV, Núm. 223, Abril-Junio 2008, 379-389

\title{
SUBJETIVIDADES SUBYUGADAS: RESENTIDOS, ARRIMADOS, HUIDIZOS, SUFRIENTES Y DESECHABLES
}

\author{
POR \\ ILEANA RodRÍGUEz \\ The Ohio State University
}

Dos preguntas entrelazadas organizan este trabajo: una concierne la relación entre teoría y prácticas sociales de investigación y su expresión narrativa; la otra, el cruce de fronteras disciplinarias. Mi punto de partida es la discusión sobre dos conceptos del liberalismo clásico, a saber, la sociedad civil y la esfera pública, con el fin de localizar en ellas las subjetividades subyugadas de "resentidos, arrimados, huidizos, sufrientes y desechables”. Mi propósito es examinar la relación entre ambos para saber si estos dos conjuntos son o no conmensurables y si, además, nos permiten hablar con propiedad de democracias y gobernabilidades. Para responder mi pregunta, leo parte de la literatura testimonial que trata la formación de estas subjetividades y estos sujetos. Tomo el caso ejemplar del fenómeno de "la violencia" en Colombia. Mi argumento fundamental es que para entender las socialidades culturales en cuestión, siempre es necesario realizar un ajuste entre teoría y práctica; esto se debe a que hacemos uso de conceptos bien establecidos y los llevamos a prácticas sociales y culturales que no responden plenamente a los mismos. Por lo tanto, el concepto siempre queda corto y el excedente de lo real sin acceso a la teoría.

Entre los testimonios producidos en Colombia a fines del siglo pasado, he elegido los de Alfredo Molano para hablar en este trabajo. ${ }^{1}$ Molano ha escrito sobre los momentos de la violencia colombiana que, con palabras de Sofía Espinosa, una de sus testimoniantes, son tres: "primero [la de] por allá con don Rojas; después me parece que en el año 65, y ahora [1970s] ésta” (7). La primera se define por la lucha entre liberales y conservadores y tiene a los campesinos sin tierra, huidizos y arrimados, como aliados de los liberales. La segunda se articula en torno al pacto entre liberales y conservadores y el despegue de una parte de los campesinos y sus aliados que se van a las guerrillas. La tercera es una lucha entre tres fuerzas, la de los libero conservadores, la de las guerrillas y las del narcotráfico. En esta presentación me limito sólo a aspectos de la primera y la segunda.

Para empezar observo que, antes de iniciar el relato de los testimoniantes, Molano advierte que lo que él ha puesto sobre el papel son historias de vida, historias, dice, "donde todos los colombianos podríamos reconocernos” (11). William Ramírez Tobón, uno de sus prologuistas, por su parte, respalda esta inflexión de totalidad al relatarnos que, en esta

\footnotetext{
${ }^{1}$ Véanse Molano, Trochas y fusiles; Los años de tropel; Mujeres de fuego. Nostalgia de la calle, o Los bombardeos en El Pato.
} 
forma testimonial, el autor "funde una o varias percepciones individuales [...] selecciona las experiencias [...] opta por unas y no otras historias” (13). Con esto, tanto el autor como el prologuista intentan sustraer la idea de que ésta es una experiencia individual pero a la vez suscribir lo que en el lenguaje de los politólogos sería traducido, por una parte, como la creación de bloques nacionales populares; y, por la otra, como el borrón de las estrictas líneas divisorias entre disciplinas, relatos y teorías. Este gesto denota "la flexibilidad de un tratamiento que permite involucrar elementos sociológicos, históricos, antropológicos y de la literatura dentro de algo tan valioso e impresionante como es el transmitir, sin mayores mediaciones aparentes, únicas e irrepetibles experiencias de vida” (14).

Dicho de esta manera, lo testimonial no está referido a un contexto explicativo externo. No integra lo particular a lo universal, sino más bien "puede convertirse en ese espejismo generoso que le ofrece aparente satisfacción a no importa qué sed y ansiedad circunstanciales” (15). Dejo colgada aquí la idea de las mediaciones que retomo en otro lugar, pero reúno estas observaciones primero porque, como ya dije arriba, mi preocupación es doble: una, la de la relación entre teoría y prácticas sociales de investigación y su expresión narrativa; la otra, la borradura de las fronteras disciplinarias. Estas dos inquietudes contribuyen tanto a la formación y reformulación de lo que constituye la nación, lo nacional y los parámetros con que se los juzga, como a dilucidar problemas relativos a la violencia dentro de las formaciones sociales.

Desde luego que en los testimonios de Molano se entrecruzan la historia política y económica de Colombia en el siglo pasado, y que una y otra componen la materialidad con la que se organiza lo social y lo personal. Esto es: ambas contribuyen a la formación del sujeto como nacional-popular. La convergencia de datos apoya las formaciones disciplinarias, pero lo esencial del relato es cómo la fluidez de "la vida" quiebra o interesecta sus fronteras. La vida, lo vivido, lo vivencial, es la categoría maestra que organiza el relato, y éste sustrae la voz popular de la abigarrada red del registro de lo legal que la excluye, para relocalizarla en una dimensión privilegiada que le otorga el estatuto de verdad y concede al hablante el precepto de sujeto de derecho. En estos testimonios la voz popular circula aparentemente sin restricciones aunque quizás el cambio de tema indique la ruptura de una palabra y el comienzo de otra. En el caso del testimonio de Sofía Espinosa, por ejemplo, podemos sugerir que el "sí, sí señor” o el "no, no señor” o el "no fue así, déjeme que le explique”, señala un quiebre que suscribe una interferencia, ya sea ésta el momento en que la pregunta del entrevistador, editada en el relato, emerge como duda, oposición, aclaración o acuerdo en la voz misma del relator que debate al enmendar o corroborar la percepción. La popularidad de la palabra está marcada por la propia inflexión regional que refuerza el afán del trazo minucioso de geografías minúsculas: Vegalarga, Algeciras, Municiones, Neiva, Florencia, Las Perlas, Tolima, Urabá, Anari, San Vicente. La geografía misma explica los giros locales, expresiones como "lo tengo muy concentrado" por "lo tengo bien entendido”; “corriendo empavoridos” por "despavoridos”; "guarecer la vida” por "sobrevivir”; “amojonado” y "melancoliado" por "tristes", etc., que van dejando su rastro dialectal regio-nacional por el texto. Es un español de malhablados muy bien narrado.

En este testimonio no es menester imaginar el registro de la voz ni hacer notar el rastro del desconocimiento de las formas. El sujeto cuenta su cuento al amanuense que lo va a transcribir con pocas enmiendas. En este sentido, un testimonio como Trochas y 
fusiles, pongamos por caso, "no es una historia de las FARC” (15), sino una historia en la que las FARC son también:

\begin{abstract}
las trochas abiertas en una descomunal y heroica empresa de movilización social a través de las cuales transitaron poblaciones enteras en un forzado proyecto de civilización alternativa de grandes sectores de nuestra geografía nacional. Son [...] una cultura marginal y [...] muy importante, acumulada a lo largo de más de medio siglo de enfrentamientos campesinos [...] detrás de sus fusiles, hay una vasta red de tejidos sociales y costumbres políticas que no se pueden encerrar dentro de las estrechas fórmulas aplicadas hasta hoy por los negociadores de paz del gobierno. (19)
\end{abstract}

Lo descomunal y heroico de la empresa, la magnitud de la movilización social y el proyecto de civilización alternativa es lo que une a los testimoniantes, a Molano y sus prologuistas, y a mí en un solo proyecto de indagación cultural, y lo que da cuenta de la construcción de subjetividades subyugadas referidas como resentidos, arrimados, huidizos, sufrientes y desechables.

¿Pero qué es lo que de estas observaciones me llama y hace eco? Resuenan primero al discutir el desfase entre teoría política y formación social. Leemos las formaciones sociales siempre ajustándolas a los conceptos centrales y mi propósito es discutir cómo esta licitación teórica denota ya una violencia epistémica, pues impide entender con propiedad los acontecimientos y explicar, en este caso, el gran fenómeno social conocido como "la violencia”. ${ }^{2}$ Es claro que desde las primeras páginas de estos textos constatamos el ejercicio de formas de violencia en cascada. La primera evidencia la constituyen las formas del salario que vienen apareadas a las formas de tenencia de la tierra; la segunda, la fundación de subjetividades subyugadas, ese vivir de fiado, de arrimado, o huyendo; la tercera, la imposibilidad del ejercicio público de la discusión sobre problemas sociales; y la cuarta y última, el ejercicio de las prácticas carcelarias y las intervenciones del ejército. Esta es una acción estatal que busca obstaculizar todo intento de los públicos campesinos para manifestarse en la esfera pública y construir aquellas instituciones de la sociedad civil que erradicarían efectivamente las formas de la violencia social. Leer estas situaciones contra el marco de las prácticas hermenéuticas del liberalismo clásico es un sin sentido. Pero no lo es percatarse que el empuje campesino de autodefensa es lo que verdaderamente ilustra la formación de una sociedad civil y una esfera pública en Colombia. De haber podido seguir su curso lógico, las comisiones, ligas y juntas campesinas podrían haber evitado la formación de subjetividades lastimadas y las políticas del resentimiento que son su corolario y dan cuenta de la violencia en el país. Para ilustrar el caso, tomo como ejemplo el testimonio de Sofía Espinosa.

DERECHO DE GENTES: INTELIGENCIA, SABER, INSTRUCCIÓN

"Yo soy fea pero no tonta” (8). La voz de Sofía Espinosa o Sofía Albarracín -mujer huidiza, sin tierra, desechable, resentida localizada en estas geografías minúsculas de fincas,

\footnotetext{
2Esto es lo que constituye la "colonialidad del poder” según lo plantea Mignolo y el "eurocentrismo" de acuerdo a Chakravarty.
} 
fundos y parcelas- es decidida y clara. Habla sin pelos en la lengua, y es dicharachera y malhablada. Por ella conocemos los sobrenombres de los agentes de la represión: los chulos, los chulavitas, los godos, los secretos. También sabemos lo que es hacer la picardía (violar a una mujer), hacerle perjuicio, hacerle el mandado, hacerle un hijo. Y que la chusma son los guerrilleros. Sofía exhibe su verdad pero lo hace dentro de un concepto claro del derecho. Cuando la capturan, sabe que no ha hecho nada y lo dice: "yo no les debía nada, yo no he robado, yo no he matado" (7). Esta conciencia del derecho de gentes que le asiste y que reitera a lo largo del relato aludiendo a que es "cristiana”, no perro; gente, no animal, se relaciona con una declaración de inteligencia que es la que quiero compartir con ustedes.

En primer lugar he de decir que el enunciado registra un alto grado de agravio, de subjetividad resentida. Hay evidencia de una profunda injuria y rabia, pero también una voluntad de defensa contra la injusticia y el miedo. De esta manera, la informante se presenta a sí misma en su doble estatuto como persona pública, sujeto de ley, y como persona privada cuya subjetividad ha sido lacerada. Si en el primer plano, el relato es una declaración de inocencia que denota de inmediato la urgencia de contar su cuento, de que se sepa esta historia; en los segundos, terceros, cuartos planos, se trata de una historia social de mayor envergadura. En la forma, llama la atención la abundancia de oraciones exclamativas e interrogativas: unas sirven de vehículo a la expresión de la sensibilidad, otras abren la puerta al discurso de la razón en la discusión pública de lo vivido. Así, exclamaciones e interrogaciones se duplican reiteradamente y testimonian en sus dobleces múltiples acusaciones: las que el ejército le hace a ella (acusaciones registradas como falsas); las que ella le hace al ejército (acusaciones registradas como verdaderas). En el proceso de la argumentación y el alegato emerge un yo autoconstituido que acusa. De este modo, mientras la exclamación declara una indignación ante la arbitrariedad y denota una posición, la interrogación se pregunta el porqué de las cosas. La razón tiene que dar cuenta de cómo el gobierno hace un travesti de la ley. Al mismo tiempo, las interrogaciones editadas que proceden del recopilador y son asumidas por el hablante sirven no sólo, como dije arriba, para ensamblar los relatos que constituyen la totalidad de la voz común, sino para aclarar, defender, explicar opiniones adversas a la circunstancia del testimoniante. Es claro que la testimoniante tiene clara conciencia del papel del recopilador que le sirve de vehículo para contar su cuento. La relación entre sujetos campesinos e investigadores letrados subyace a las relaciones disciplinarias.

Insisto en la declaración de honradez y de legalidad de la que da fe el sujeto -"Yo no les debía nada, yo no he robado, yo no he matado"- como manera suya de delatar las arbitrariedades de una gobernabilidad que hace un travesti de la ley, pero mi interés particular es encabalgar honradez y sabiduría. La oración "soy fea pero no tonta”, hace referencia a un entender, a un inteligir que es a la vez conocimiento de las cosas y conciencia de las cosas: “realización, darse cuenta o percatarse de". Este "percatarse de" es inevitable pues nace en el cuerpo mismo, de ahí que la vida y lo vivencial sean las propuestas sobre las cuales se predica la verdad como conocimiento y, aun más, como certeza. Pues es la carne misma la que conoce y experimenta la verdad y la mentira, y los criterios de juicio dicen que "verdad" es que el ejército enciende los ranchos, acaba con las cosechas, viola mujeres, mata hombres, persigue e imposta a los guerrilleros, tergiversa a propósito las formas de la organización popular. El “sí señor”, “no señor”, van marcando los hitos verdad/mentira. 
Ejemplos de esta escisión verdad/mentira pueden encontrarse en la tematización que va delimitando los campos del saber campesino. El campesino conoce en primer término la tierra, su importancia y su cultivo; conoce la política, las divisiones partidistas, las tácticas y estrategias de unos y otros, los campos de fuerza y sus fuerzas. Además, conoce a fondo su propia sensibilidad y en última instancia, conoce que conoce. De tal manera están unidas sabiduría y represión, sabiduría política y sabiduría agrícola, que esto es lo que da lugar a la subjetividad lastimada que declara la escisión entre cristiano y perro. Es más, el saber refiere a una hermenéutica que sirve para ordenar una serie de actos que constituyen patrón y hace sistema. Lo más importante de este sistema para el sujeto que lo capta inmediatamente es que su vida biológica, natural, está amenazada por ello. Por eso la vivencia se traduce con claridad en el sistema simbólico y se convierte en condición de saber, en certidumbre. Para el recopilador que capta esa vivencia, el cruce entre vida biológica y vida como componente disciplinario se entremezclan ineludiblemente.

Para mí el informe no sólo constata el lugar desde donde la convergencia de datos mezcla el saber disciplinario que luego se desglosa, sino que se convierte en instancia no tanto de una realidad sin teoría como el lugar de la catacresis, en el sentido de la mala aplicación de un término o la substracción del mismo de su significación propia. Pues es en el relato de vida tanto como en la vida misma del informante donde encuentro la concurrencia de una teoría cuya praxis está subyugada. Es ahí, en la vida de los sujetos campesinos y sus prácticas, donde se encuentra el terreno en el que convergen teoría política, prácticas y formaciones sociales liberales. Esto se entiende claramente en el alegato de Sofía y en las instancias de su tematización. Es la tematización la que pone al alcance del lector el saber campesino.

No tengo tiempo aquí de entrar en la relación entre sabiduría, conocimiento y política, pero baste decir para terminar que desde una posición de saber en el “saber” de gentes, Sofía reconstruye su idea del sistema de tenencia de la tierra que entronca muy bien con el de los otros personajes de Molano. Es este sistema el que le da a Sofía la base para constituirse como sujeto de ley a partir de su entendimiento del derecho. Así, la frase "Yo soy fea pero no tonta” significa que dentro del aparato que juzga a "la mujer”, ella como campesina no posee los encantos físicos deseables -juicio que acepta-, pero esta carencia de atractivo físico no se traduce en la incapacidad de saber, en ser tonta. Tonta es un adjetivo que se refiere a los niños, a quienes llama "tonticos". El "tontico" habla por chico y por miedo. Pero ella sabe y lo que sabe es "que la única seguridad es medio tener un puchito de tierra” (11). Termino, entonces, citándola: "No señor, uno también es cristiano, a uno le faltará mucha cosa de cultura, o de inteligencia, pero deberíamos ser más atendidos por el gobierno, porque la pobrecita campesina es la más desprotegida y la más importante... Somos tontos, pero tenemos el pleno conocimiento que si no hay campesinado no hay pueblo que se mueva” (20). Ese cambio de planos enunciativos establece un saber ligado a la supervivencia, pero también al sentido de lo político y de lo público.

Hay varios asuntos a dirimir aquí. El primero es que el saber y el conocer están ligados a un secreto -como en Rigoberta- que de alguna manera pone a la persona que lo posee en riesgo de delinquir. Aunque callar es un derecho, no hablar es delincuencia. No decir, ocultar, significa no querer identificar, desconocer. Por un perverso juego de la significación legal, significa también “creer en”, y el salto de “saber” a “creer” cobra una cierta veracidad cuando 
la testimoniante usa como sinónimos “militar” y “participar” para referirse a su participación en la Junta. También resulta que el quiebre del relato, el no seguir el hilo y pasar a hablar de otras cosas es también sintomático de un guardar el secreto. Esto es, o guarda el secreto o no lo desconoce. En esta ambigüedad se coloca el relato. No obstante, en el cambio de planos es posible notar el ensamble de las historias de los informantes que Molano usa para constituir su "personaje" colectivo y así completar con el relato de varios una sola visión de la cosa. Pero al mismo tiempo es evadir la pregunta sobre qué es lo que sabe Sofía de la gente, de la chusma, de los guerrilleros. Puede formularse la hipótesis de que los hitos de transición, donde termina un informante y empieza otro tienen que ver con el propósito que alienta a Molano a recopilar la información, y ese propósito se abraza con lo que arriba indicamos, esto es, las grandes movilizaciones campesinas, los grandes éxodos causados primero por el odio a muerte entre liberales y conservadores y luego por la alianza de estos con el propósito de borrar la insurgencia guerrillera. Y aquí es donde la sinonimia entre militar y participar, tanto como el nombrar a los guerrilleros "chusma” y "los muchachos", y llamar a los miembros de la Junta campesina “compañeros”, podría marcar los desfases que el cambio de interlocutores facilita pues le permite al recopilador seguir el hilo de la historia que le interesa narrar y al mismo tiempo excluir la información sobre si el informante posee realmente información sobre los guerrilleros o no.

En el primer corte, Sofía se dirige directamente al interrogador, al entrevistador, y explica el significado que para ella tiene la geografía. Ser de El Pato, tanto como el sentido de distancia que marca “cerca de” y "lejos de”, significa tener una familia, una parcela, y significa también "no ser tonta”, sin luces, nena, menor de edad. Esta conciencia de la mayoría de edad, que es madurez de juicio, viene aparejada al conocimiento y a la certeza de su circunstancia personal que emana del conocimiento de su propia geografía y de cómo ésta significa familia, comida. Es en este momento cuando el saber de gentes se torna saber geográfico, saber agrícola, y saber político, los tres grandes saberes de los que habla el texto por boca de campesinos sin tierra, huidizos. Va a la tierra, a la parcela, porque esa es su manera de significar si no su nación, al menos su lugar, su pedazo de tierra, su local y arraigo que reclama para sí y su familia. Por eso es que al intento de localización exacta, cerca de qué y lejos de qué, responden la serie de nombres que se localizan en los entornos. Esta es su manera de saber nombres, de saber (identificar) gentes. La geografía y el lugar de la localización resultan claves. Es el sitio desde donde se enuncia la verdad, verdad que se repite en otro sitio pero que se genera en el primero. La geografía también marca una peregrinación que va de un lugar a otro en busca de o huyendo de, y que convierte al sujeto de ley en sujeto ilegal, huidizo, errante. Los nombres son significantes en la medida que marcan sitios minúsculos, microscópicos, los poros de la nación y de la nacionalidad.

Tres cosas son importantes en el relato de Sofía: primero está el saber/conocer ligado a la tierra y el entorno del que hablamos; segundo, el del funcionamiento de las Juntas; y tercero, el del funcionamiento del ejército. Si en el primer relato lo que tenemos es la constitución de regímenes de subjetividad, en el segundo lo que encontramos es la conciencia de cómo se opera en la esfera pública; y en el tercero, la clara conciencia política donde el conocimiento de gentes viene a ser sólo el corolario del conocimiento de sí y la instancia certera de las dos fuerzas que operan en una guerra de posiciones en la que ninguno gana. Quiero relevar 
aquí el segundo momento que se refiere a la conciencia de cómo operar en la esfera pública porque esto ensambla con el relato de Yosa y me permite concluir este trabajo. Cuando Sofía habla de las Juntas su testimonio se relaciona con la discusión sobre las comisiones y ligas campesinas.

El relato de la Junta está organizado en torno a tres ejes: la honradez, la gobernabilidad, y la noción de totalidad. Sobre estos pilares se construye la noción de gobernabilidad democrática. El signo “todos", reiterado, constituye un camino y marca la ruta a seguir en el mapa donde la meta es el bien común. Dice: "porque la Junta es la única autoridad por allá. Todos militamos en ella. Como un decir: todos participamos en ella... todos vivimos de acuerdo a lo que la Junta diga, cualesquiera cosa que se necesita, entonces nos llama la Junta... Entonces nos ponemos de acuerdo y se hace. También la Junta hace pedidos, peticiones... Entonces la Junta toma un acuerdo, una solución para todos, para bien de todos...” (11). La Junta es autoridad, gobierno, sociedad civil y esfera pública, esto es, la mejor manifestación del gobierno de gentes. Para dirigir la Junta hay que ser honrado. La honradez es la condición de posibilidad de la confianza y fundamenta la responsabilidad en el buen servicio. La junta es un lugar de participación social en el que todos se ponen de acuerdo, en el que todos hablan, en el que todos participan: "Nosotros tomamos el acuerdo en votación, a la vista de todos. Nos reunimos y votamos. Todo mundo puede manifestarse, decir, hablar lo que quiera sin ofender” (13). La Junta también es un punto de contraste entre otro tipo de organizaciones sociales, políticas. Difiere de la tropa y de los guerrilleros. La distancia que establece respecto a estas otras dos formas de socialidades nos ayuda a pensar en una especie de tercera fuerza representada por el campesinado que lo que quiere es trabajar. Es mediante el trabajo que se sobrevive y es de nuevo la sobrevivencia, la defensa de y responsabilidad por la vida, la que constituye la civilidad, la civilización y la comunidad. Eso es lo que decía Hegel al hablar del tránsito de la sociedad natural a la civil y de ella a la política. Que era el trabajo, considerado en abstracto, lo que facilitaba el tránsito entre lo particular y lo universal que constituía el bien común.

Claro está que hay cabos sin atar. La palabra “militar”, por ejemplo, hace ruido en el sistema pues denota un lenguaje que llama la atención sobre el tercer tema de este testimonio que es la relación entre guerrilleros/ejército y gente. Parte del recuerdo de Gaitán y de las luchas entre liberales y conservadores para hablar del presente y cuenta un cuento que es una vigente pues nos habla del valor literario del relato: “A los hombres les hacían la caphorca...: los empelotaban y los amarraban con una cuerda de triple. Una punta en la garganta y la otra punta en las nobles, y las apretaban bien, que no corriera; la apretaban así a quedar la boca al pie de las güevas, quedaban bien acurrucados. Entonces les pegaban una puñalada aquí, al lado de los riñones y al sentir la puñalada el cristiano se enderezaba, levantaba la cabeza y de una vez: se ahorcaba y se capaba” (15). Este pequeño relato habla con suficiencia del sentir de la gente y justifica, si se quiere, ese desconocer el “saber de gentes”. Ellos saben que los dos ejércitos pasan por el lugar y que a los dos hay que darles de comer, compartir con ellos. Pero el problema es que a veces el ejército se disfraza de guerrillero y cuando le dan de comer, dice que la gente apoya las guerrillas. Este simulacro o mentira también despierta la conciencia de la gente en cuanto a las trampas que les tiende el gobierno. Y dice, con razón, que "para pelear se necesitan dos" (18) y que esos pleitos los hacen vivir en la zozobra. Con estas palabras cierro el testimonio de Sofía para abrir el de Yosa que se ocupa del primer momento de la violencia pero del cual no me ocupo en esta presentación. 
El testimonio de Isauro Yosa, el mayor Lister de las FARC colombianas, narra la historia de la migración forzada de los sin tierra, los arrimados en el ámbito geográfico de una comarca colombiana. La minuciosidad y el detalle con el que se nombra esta geografía desconocida es un hito que hay que comentar, como lo hago más adelante. El testimoniante sale de esta geografía por una ambición de juventud: vestir bien. Este deseo juvenil, que el testimoniante compartirá con los sicarios en los testimonios de Alonso Salazar y las novelas de Fernando Vallejo, termina en la organización de las FARC. Me detengo brevemente en el principio del testimonio porque ahí es donde Yosa habla de la formación de las comisiones (campesinas), de las ligas, en las cuales se ponen en evidencia los intentos de formación de la sociedad civil colombiana. La segunda parte del testimonio habla de la formación de las guerrillas como resultado de la derrota de la representación civilista. Así pues, la narrativa sirve de archivo a la dinámica que articula la radicalización de un movimiento civil y democrático, y atestigua la inventiva de la gente en formar instituciones que vehiculen sus discusiones, consensos y acuerdos dentro del panorama nacional.

El contexto político mayor se define por la lucha de liberales y conservadores (godos) en la cual se inserta la de los trabajadores del campo sin tierra. Su libro Los años del tropel. Relatos de la violencia va a magnificar este otro trozo de historia colombiana. Pero en Tropeles, las luchas entre liberales y conservadores introducen una narrativa económica en la que se pone en evidencia un sistema laboral que es desleal al trabajador y que se manifiesta alrededor de una sociabilidad basada en el engaño. El trabajador se coloca en una hacienda que paga, en 1926, “cinco centavos diarios” (23) por recoger café y que obliga a la gente a vivir de fiado. Por ejemplo, Yosa se casa porque le "prestaron quinientos pesos para pagar en trabajo. Terminé de pagarlos [dice] cuando los niños hicieron la primera comunión” (24). Esta situación de perpetua deuda se agrava cuando sabemos que el sistema de pago está basado en el fraude: "La arroba de café que uno trabajaba no era de quince medidas sino de doce, pero la arroba que uno compraba en el comisariato de la hacienda no eran quince medidas, sino dieciocho. Así nos daban por la cabeza dos veces” (25).

La historia de Yosa empieza en la pobreza de los arrendatarios. Los sujetos que hablan son medieros, colonos, arrimados: “don Ricardo Trujillo... no dejaba de recordarle todos los días a mi papá que éramos arrimados” (22), los que terminan engrosando las filas de las FARC. Esta es una historia que cubre todo el siglo pasado pues Yosa nace en 1910 y hacia 1950, cuando tiene cuarenta años, se las ingenia para organizar una coalición de defensa armada con un hacendatario liberal. Ahí es donde empieza la segunda etapa de la violencia. La coalición liberal-popular es el marco contra el que se recorta el relato y cuyo punto alto está representado por el señero nombre de Gaitán: Jorge Eliécer. Digamos entonces que el testimonio publicado en la serie de Historia Contemporánea del fondo editorial CEREC, CINEP, y estudios rurales latinoamericanos tiene como primera intención construir el archivo de la voz popular para que esta misma cuente su historia. Historia y cuento, esto es, historia y literatura son los aliados en la formación de los regímenes de conocimiento que van a constituir los nuevos regímenes de subjetividad nacional-local. Y a esto responde en primera instancia la salida de los marcos teóricos preestablecidos. Es decir que la palabra del sujeto histórico local desmiente desde luego ciertos rasgos de la apreciación teórica. Esto es lo que Julio Ramos, hablando de la esclava Maria Antonia, va 
a caracterizar como una instancia de "la relación profunda entre el derecho al testimonio y la historia del concepto de la ciudadanía [...] la enunciación de la verdad en un testimonio era una actividad definitoria de la ciudadanía” (43).

Desde los dieciséis años de vida (1926) converge en este sujeto o sujetos -una misma historia se cuenta a varias voces- la historia laboral ligada a los sistemas de tenencia de la tierra que articulan todas las violencias señaladas arriba. Esta no es sino la historia otra, el punto de vista que dialoga con la historia oficial y legal constituida por las narrativas desarrollistas (Escobar, Encountering Development). Este testimonio pone al descubierto cómo, de haber tenido fuerza la voz campesina, el desarrollo del capitalismo agrario hubiera sido una de las dinámicas de los procesos productivos y de acumulación de capital en Colombia. O sea que si lo que está en cuestión son las teorías del desarrollo o la democratización, en estos testimonios asistimos a la razón social que obstaculizó ambas. En la materialización de esa voz de colonos, medieros, arrimados, descansa nada menos que la formación de un desarrollo alternativo junto a la de la formación del bloque nacional popular que lideró en su momento Jorge Eliécer Gaitán.

El corolario tiene gran significación en la medida en que "la violencia” campesina, entendida como autodefensa de vida, por una parte, y como contribución a la discusión racional en la esfera pública, por otra, contrapone una visión alternativa de desarrollo, paz social y democracia en Colombia al menos desde principios de siglo. Porque, ¿qué es lo que hacen los campesinos en la primera mitad del siglo? Empiezan discutiendo un dato: la inexactitud de los pesos y medidas que rigen el trabajo en las balanzas que pesan el producto. Primero se dan cuenta del fraude y a propósito de él, para contrarrestarlo, convocan asambleas, las que a veces se reúnen los fines de semana o en la tarde para dar lugar a la mayor representatividad. Estas asambleas discuten el derecho de gentes y constituyen las comisiones o ligas, una tras otra hasta llegar a diez: "Las diez comisiones formaron al poco tiempo lo que se llamó una liga campesina, y ya con nombre [afirma el testimoniante] más nos oía la gente y más nos odiaban los patrones” (26). Poco a poco van ganando representatividad y ganan "para Gaitán dos bancas en el concejo" (26).

Hasta este momento lo que vemos en movimiento es primero la constitución de un sujeto social con derechos. El testimonio de Sofía Espinosa, titulado “Testimonio de Sofía Espinosa”, recoge esta preocupación por los derechos civiles de las personas con más exactitud. Sofía es la que mejor articula este derecho de gentes. Ella se refiere a la inteligencia campesina como sabiduría y establece una distinción entre la inteligencia, la tontera y la instrucción letrada. La tontera es niñería y es minoría de edad. A los niños les llama tonticos. En otra parte hablo de este asunto en más detalle.

En Yosa asistimos también a la constitución del sujeto social como sujeto de derecho. Este testimoniante nos informa que "las ligas dieron una nueva orientación: rozar para sembrar, sin respetar las tierras en montaña que las haciendas reclamaban. Hacíamos comisiones de cincuenta o de cien hombres para ir a trozar monte, hacer rocerías y sembrar. Al comienzo la policía nos sacaba, hasta que se le perdió el respeto a la autoridad” (26). Desde el principio vemos cómo los campesinos construyen el sentido a través del consenso en sus organizaciones y cómo la lucha se organiza a través de la defensa del trabajo que contradice los deseos y planes de los hacendatarios. 
El litigio comienza con el alegato sobre la pertenencia de la tierra, si las tierras realmente son de sus dueños o no, pues sabemos que en muchos casos estos orígenes provenían de la desposesión de tierras comunales a los indígenas, de escrituras de 1840 en las que se hablaba de “compras”, pero sin delimitar exactamente de dónde a dónde o de dónde hasta dónde se compraba y cómo: "La finca de don Camilio Iriarte tenía unas escrituras de 1840, en las que se reconocía la compra de una mejora de dos hectáreas [...] La compró con trapiche, casa y dos bestias a un indio, pero cuando nosotros investigamos el caso [...] nos dimos cuenta [...] que el globo de tierra [...] tenía cien mil hectáreas” (24-25). Así las cosas, la cuestión de la legalidad o ilegalidad de la tenencia de la tierra de los hacendatarios se cruza con la discusión sobre la legalidad o ilegalidad de cultivarla por parte de los arrimados, sin tierra. Vale preguntar quién es aquí el sujeto de derecho. ¿A quién asiste la razón en esta discusión pública? ¿Qué tribunal puede establecer o legislar la verdad? El problema real es que en estos menesteres, en la relación entre campesinos y hacendados no hay debate sino solo confrontación, una forma de violencia legalizada y apoyada por la fuerza del ejército -los chulos, la chulamenta, los pájaros; violencia vertical que al acelerarse alcanzará la forma de una violencia horizontal.

Es más, al agarrar fuerza las ligas campesinas, la enunciación cambia de signo y en vez de llamar liberales a sus miembros, les empiezan a llamar comunistas. El nombre marca el desliz de una alianza a otra, de una coyuntura a otra, y, posiblemente, de un testimoniante a otro. Lo más grave es que la verdad se desliza de un sistema de validación a otro y se escabulle y enreda en las normas de representación significadas por el cambio de nombre -de sin tierra a comunista-. Un cuerpo teórico otro, una hermenéutica alterna emerge en el acto mismo del habla al cambiar de persona el informante. La resolución del conflicto se posterga y se confunde. O sea que el intento de organización campesina, de hacer audible la voz intersecta la lucha global de los trabajadores a quienes más temprano que tarde se les hará los únicos responsables de la violencia. Y luego se hipoteca el sentido al convertirlos en los enemigos de la democracia.

Pero bien visto, son los trabajadores en su lucha por dejar oír su voz y en sentirse con derechos a constituirse en lo que Nancy Fraser llama contra públicos subalternos, los mismos que en sus asambleas, en sus acuerdos y consensos, representan la verdadera idea de la democracia, esto es: encarnan los principios teóricos del liberalismo clásico escritos en los libros de Hegel, Locke, Smith. En otras palabras, sus prácticas sociales son conmensurables con las prácticas teóricas. Son ellos los que al representar el bien común mejor encarnan la idea de la gobernabilidad democrática, lo que Charles Taylor argumenta como la voz pública trabajada en la esfera pública a la que el gobierno responde y representa. Es claro que el triunfo de las ligas era el triunfo del capitalismo agrario en Colombia. La ley de Tierras, emitida en 1936, la famosa ley 200, se apegaba a lo que los campesinos sin tierra deseaban.

La guerra por las tierras, por la parcelación, es la guerra por el sentido, por la significación de la nación. La base del problema es el acaparamiento de tierras que se traduce en un acaparamiento de la ley y del sentido. Consecuentemente, la discusión pública en lugar de conducir a la democratización conduce a la radicalización. Y esto sucede porque a las tácticas civilistas de los sin tierra se les responde no con la discusión sino con la fuerza. Es esta una dinámica que radicaliza simplemente porque a la democracia se le ponen obstáculos. Los 
sin tierra y los con tierra hablan dos idiomas diferentes, de dos referentes diferentes, aun si ambos hablan del trabajo. Los unos quieren labrar para comer y para sobrevivir; los con tierra quieren riqueza fácil. Así el trabajo y la tierra obedecen al mismo sistema desreglamentado en el que el trabajador es engañado y frente al cual ingenia formas alternas. Cuando estas fallan y al sujeto se le orilla, éste se va a la guerra, a la delincuencia, al crimen, y al terror. Este es el final de la etapa de la razón discursiva y el comienzo de la razón armada, el fin de la lucha discursiva por la democracia y el principio de la fuerza que se opone a la fuerza.

El segundo momento es la alianza armada entre hacendatarios liberales y los sin tierra que hasta ahora son liberal-comunistas. Así empieza la historia de la nación dividida, la movilización de poblaciones, la construcción de campamentos, la distribución del trabajo, esto es una sociedad alterna dentro de la sociedad. Dice Yosa: "Una vez construidos los alojamientos [...] ubicadas las ranchas, instalada el agua y destacadas las comisiones armadas o guerrillas [...] había que organizar la comida [...] se nombraron comisiones para ir a trabajar, para tumbar monte y hacer cosechas [...] Más de doscientos hombres trabajaban en esa meta” (36). La tropa se forma a partir de una serie de tipos sociales cuyos apelativos revelan su origen social: Arboleda, Santander, Jalisco, Tirofijo, Charro Negro; a algunos, como Marín, se les describe “cerrado de cara, poco amigo de chanzas” (37), otros como “fogoso, avispado, ágil para pelear, inquieto" (37). De esta primera etapa sale la idea de que los liberales se dividen en limpios (los del directorio) y comunes (los comunistas). Con la formación de las FARC se cierra la etapa de la lucha civil.

\section{BiBLIOGRAFÍA}

Escobar, Arturo. Encountering Development. Princeton: Princeton UP, 1998.

Molano, Alfredo. Mujeres de fuego. Nostalgia de la calle, o Los bombardeos en El Pato. Bogotá: CINEP, 1978.

Los años del tropel. Bogotá: CEREC-CINEP, 1985.

Trochas y fusiles. Bogotá: IEPRI, 1994

Ramos, Julio. Paradojas de la letra. Caracas: eXcultura, 1996. 